\title{
Nota sobre la dieta del gavilan planeador Circus buffoni (Aves: ACCIPITRIDAE) en el valle de inundación del Río Paraná, Argentina
}

Beltzer, Adolfo H.'; Tomatis, Maria F.2; Díaz, Héctor F.3.

(1) Investigador del CONICET. Instituto Nacional de Limnologia (INALI-CONICET). José Maciá 1933, 3016 Santo Tomé: Santa Fe, Argentina. e-mail: inali@ceride.gov.ar

(2) Facultad de Humanidades y Ciencias. Universidad Nacional del Litoral (UNL). Paraje El Pozo, Santa Fe, Argentina. Pasante en el INALI.

(3) Estudiante de Biologia. Facultad de Ciencia y Tecnologia, Universidad Autónoma de Entre Rios (UADER). Paraná. Entre Rios. Argentina.

RESUMEN: Se dan a conocer los resultados del análisis del contenido estomacal de tres individuos de Circus buffoni capturados en la isla Carabajal (Santa Fe) y una observación. Los resultados permiten ampliar el conocimiento del espectro alimentario de la especie para el área, incorporando aves(Agelaius cyanopus) roedores de la especie Holochilus brasiliensis e insectos coleópteros (Curculionidae y Carabidae).

Palabras clave: Aves, Circus buffoni, dieta, rio Paraná.

SUMMARY: Note of the diet of Long-Winged Harrler Circus buffoni (Aves: Accipitridae) in the Middle Parana River Floodplain, Argentina. Beltzer, Adolfo H.; Tomatls, Maria F; Diaz, Héctor F.. The results of three stomachs contents of LongWinged Harrier Circus buffoni collected in Carabajal island (Santa $\mathrm{Fe}$ ) and one observation were described. This study allow us to increase the specie alimentary spectrum knowledgement for this area, including birds (Agelaius cyanopus), Rodentia (Holochilus brasiliensis) and insects Coleoptera (Curculionidae and Carabidae).

Key-words: Birds, Circus buffoni, diet, Paraná River. 